\title{
Study of DNA methylation patterns of imprinted genes in children born after assisted reproductive technologies reveals no imprinting errors: A pilot study
}

\author{
HAI-YAN ZHENG ${ }^{1 *}$, XIAO-YUN SHI ${ }^{1 *}$, LE-LE WANG $^{1}$, YA-QIN WU ${ }^{1}$ SHI-LING CHEN ${ }^{1}$ and LIN ZHANG ${ }^{2}$ \\ ${ }^{1}$ Center for Reproductive Medicine, Department of Obstetrics and Gynecology, Nanfang Hospital, \\ and ${ }^{2}$ Department of Histology and Embryology, School of Basic Medical Science, \\ Southern Medical University, Guangzhou, Guangdong 510515, P.R. China
}

Received February 25, 2011; Accepted April 26, 2011

DOI: $10.3892 /$ etm.2011.261

\begin{abstract}
Assisted reproductive technology (ART) including in vitro fertilization (IVF) and intracytoplasmic sperm injection (ICSI) have been shown to be associated with abnormal genomic imprinting, thus increasing the incidence of imprinting disorders such as Beckwith-Wiedemann syndrome (BWS) and Angelman syndrome (AS) in ART-conceived children. Furthermore, a recent study described abnormal DNA methylation in clinically normal children conceived by ART. However, data from different studies are conflicting or inconclusive. This study examined DNA methylation patterns of multiple imprinted genes in children born after ART to primarily evaluate the impact of ART on genomic imprinting. A total of 101 newborns conceived by ART (40 ICSI and 61 IVF) and 60 naturally conceived newborns were involved in our study. After obtaining the approval of the Institutional Ethics Committee, umbilical cord blood was collected from each infant. Genomic DNA was isolated from each blood sample and treated using sodium bisulfite. Subsequently, using methylation-specific PCR (MS-PCR), we analyzed six differentially methylated regions (DMRs) including $K v D M R 1, S N R P N, M E S T, M E G 3$, $T N D M$ and XIST. Meanwhile, information regarding twin pregnancies, gestational age, and birth weight of the neonates
\end{abstract}

Correspondence to: Professor Shi-Ling Chen, Center for Reproductive Medicine, Department of Obstetrics and Gynecology, Nanfang Hospital, Southern Medical University, Guangzhou, Guangdong 510515, P.R. China

E-mail: chens1_92@163.com

Professor Lin Zhang, Department of Histology and Embryology, School of Basic Medical Science, Southern Medical University, Guangzhou, Guangdong 510515, P.R. China

E-mail: zlilyzh@126.com

*Contributed equally

Key words: assisted reproductive technology, genomic imprinting, imprinting disorder, DNA methylation was documented. None of the cases presented with phenotypic abnormalities. Children conceived by ART were more likely to have low birth weight and to be born before term, compared with children conceived spontaneously. However, 7 months to 3 years of clinical follow-up showed that none of the children had clinical symptoms of any imprinting diseases. Furthermore, the MS-PCR results showed that all 161 children had normal DNA methylation patterns at six DMRs despite the different mode of conception. Our data did not indicate a higher risk of DNA-methylation defects in children born after ART. However, further studies using quantitative methods are needed to confirm these results.

\section{Introduction}

Recently, concerns about an association with assisted reproductive technologies (ART) and genomic imprinting defects have been raised $(1,2)$. Genomic imprinting is a process of chemical modification of nucleotides in which only one allele of a specific gene is functioning and the other allele is silenced based on the parent-of-origin. The most thoroughly studied epigenetic modification is methylation of CpG dinucleo-tides in cis-regulatory sequences (3). Allelic expression of imprinted genes is controlled by differentially methylated regions (DMRs) which are thought to function as imprinting control centers (4). DMRs are thought to be particularly sensitive to disruption by environmental factors such as the large amounts of gonadotropin and different culture mediums $(5,6)$.

The effect of in vitro fertilization (IVF) on DNA methylation in mouse embryos has been demonstrated in many studies (5,7-9). Small epidemiology studies in humans have suggested a 4- to 9-fold increased incidence of BeckwithWiedemann syndrome (BWS) among children conceived by IVF or intracytoplasmic sperm injection (ICSI) (10-13). The loss of maternal DNA methylation at the DMR of $K C N Q 1$ represents the most frequent alternation in BWS patients (11-13). Moreover, epimutations at other DMRs in ART children resulting in BWS, such as the mesoderm-specific transcript (MEST), or small nuclear ribonucleoprotein-associated polypeptide $\mathrm{N}(S N R P N)$ have also been reported (14). 
However, in a large cohort study from Denmark including 6,052 IVF singleton children and 442,349 normally conceived singletons, there were no cases of imprinting-related disease identified in the IVF group and 54 children with imprintingrelated diseases in the non-IVF cohort (15). An increased risk of the overall imprinting diseases after IVF was not observed in the study (15). A questionnaire-based survey from the Republic of Ireland and Central England aimed to detect children after ART with possible diagnosis of BWS or Angelman syndrome (AS) (16). In the study, data from 1,524 children were analyzed and only one case of BWS was identified having hypomethylation at KvDMR1, suggesting that although prevalence of BWS in children born after ART may be higher, the absolute risk of having a child with BWS conceived by ART is notably remote (16). Moreover, ten DMRs were analyzed in 185 phenotypically normal children by Tierling et al, and they found no association with ART and imprinting (17). Notably, in a recent study conducted by Gomes et al, aberrant imprinting was observed in 3 out of 18 clinically normal children conceived by ART (18), suggesting that the impact of ART on the epigenetics is not completely understood.

In order to investigate the possible genetic risk of DNA methylation defects associated with ART, we analyzed the methylation patterns of six DMRs in 161 phenotypically normal children, comprising 40 children conceived by ICSI, 61 by IVF and 60 conceived spontaneously. The DMRs analyzed included KvDMRI, SNRPN, MEST, maternally expressed gene 3 (MEG3), transient neonatal diabetes mellitus (TNDM) and X (inactive)-specific transcript (XIST).

\section{Materials and methods}

Study population and DNA samples. Informed consent and medical information release documents were obtained as approved by the institutional review board. Data regarding maternal age, gestational age, birth weight, and birth length of the neonates without pathological findings were documented. Umbilical cord blood samples from 101 children born after ART and 60 children conceived spontaneously were taken directly after birth and stored at $-80^{\circ} \mathrm{C}$. DNA purification was performed using 3-7 ml EDTA-blood with the Genomic DNA purification kit (Promega, USA).

Sodium bisulfite treatment and methylation-specific PCR. The methylation assay was performed at the DMRs of six imprinted genes (KVDMRI, SNRPN, MEST, MEG3, $T N D M$ and XIST). Bisulfite treatment of genomic DNA was performed with the EpiTect Bisulfite kit (Qiagen, Germany). Methylation-specific PCR (MS-PCR) utilizes this sodium bisulfite treatment to distinguish methylated from unmethylated DNA $(19,20)$.

Purified, non-methylated and methylated human DNA standard was used as the negative and positive control in the methylation detection applications, which was performed with the Human Methylated \& Non-methylated DNA set (Zymo Research, USA). Each sample was analyzed in two independent MS-PCR reactions. PCR reactions were carried out with a GeneAmp PCR System 9700. The 25- $\mu$ l PCR reaction mix contained 2X PCR HotStart Premix buffer (Takara, Tokyo, Japan), $0.5 \mu \mathrm{M}$ primer-M forward and $0.5 \mu \mathrm{M}$
primer-M reverse in the PCR reaction amplifying the methylated imprint specifically or $0.5 \mu \mathrm{M}$ primer-U forward and $0.5 \mu \mathrm{M}$ primer-U reverse in the unmethylated PCR, and $2 \mu \mathrm{l}$ of bisulfite-modified DNA. Primer sequences for KvDMRI (21), MEST (22), SNRPN (23), MEG3 (24), XIST (25), and TNDM (26) were used as described, and PCR programs were summarized in Table I. PCR products were separated on a $2 \%$ agarose gel, stained with ethidium bromide and visualized under UV illumination.

Statistical analysis. SPSS 16.0 was used to analyze the characteristics of the study population. Continuous variables were presented as the mean $\pm \mathrm{SD}$ and were tested by two-sample t-test or analysis of variance (ANOVA). The Chi-square test was used to compare the categorical neonatal and maternal data. $\mathrm{P}<0.05$ was considered statistically significant.

\section{Results}

Clinical data. Blood samples were collected from 101 children born after ART (61 conceived by IVF and 40 by ICSI) and 60 children conceived spontaneously. IVF and ICSI procedures were carried out following standard protocols (27). The ICSI was performed with ejaculated spermatozoa in the majority of the cases $(n=37,92.5 \%)$, with fresh testicular spermatozoa after testicular sperm extraction (TESE) in 1 case $(2.5 \%)$ and with epididymal spermatozoa after microsurgical epididymal sperm aspiration (MESA) in 2 cases (5\%). The ICSI procedure was performed due to male factor infertility in all couples.

A major difference between the three sample groups concerns the frequency of twin pregnancies. Due to multiple embryo transfer following ART twin pregnancies were very frequent in the IVF and ICSI groups. Of the 101 babies from ART, 46 were delivered from 23 twin pregnancies, and the remaining 55 children were born from singleton pregnancies, while only 2 twin pregnancies were among the 60 children in the spontaneously conceived group $(\mathrm{P}<0.05)$. As compared with the infants in the spontaneous pregnancy group, infants conceived by IVF and ICSI were more likely to have low birth weight and to be born before term $(\mathrm{P}<0.05)$ (Table II). In addition, maternal age was significantly higher in the IVF group than that in the spontaneous pregnancy and ICSI groups.

All 161 children participated in a clinical follow-up. None of the children (aged between 7 months and 3 years in the follow-up) had clinical symptoms of any imprinting diseases, e.g, feeding problems, reduced weight gain, mental retardation, short stature, absence of speech and paroxysms of laughter.

DNA methylation patterns in children born after ART and after spontaneous pregnancies. MS-PCR of five DMRs (KvDMR1, SNRPN, MEST, MEG3, and TNDM) from normal individuals generated two products from the methylated and unmethylated alleles of genomic DNA. Regarding XIST, where in females, the DMR is DNA methylated on one X chromosome whereas the other chromosome is unmethylated; but in males, who have only one $\mathrm{X}$ chromosome, the single locus is normally methylated, alternatively, only methylated allele can be detected. All of the 161 children born after ART and 
Table I. Analyzed differentially methylated regions (DMRs) and methylation-specific PCR procedure.

\begin{tabular}{|c|c|c|c|c|c|c|}
\hline \multirow[t]{2}{*}{ DMRs } & \multirow[t]{2}{*}{ Chromosomal location } & \multirow[t]{2}{*}{ Allelic methylation } & \multicolumn{2}{|c|}{$\mathrm{Ta}$} & \multicolumn{2}{|c|}{ Size (bp) } \\
\hline & & & M & $\mathrm{U}$ & M & $\mathrm{U}$ \\
\hline$K v D M R 1$ & $11 \mathrm{p} 15.5$ & Maternal & 60 & 58 & 170 & 170 \\
\hline$M E S T$ & 7q32.2 & Maternal & 60 & 60 & 300 & 300 \\
\hline SNRPN & $15 q 11-q 13$ & Maternal & 60 & 60 & 216 & 313 \\
\hline MEG3 & $14 q 32$ & Paternal & 60 & 60 & 160 & 120 \\
\hline$X I S T$ & $X$ & & 60 & 60 & 264 & 280 \\
\hline$T N D M$ & $6 q 24$ & Maternal & 60 & 60 & 175 & 187 \\
\hline
\end{tabular}

$\mathrm{U}$, unmethylated allele; M methylated allele. The polymerase was activated at $95^{\circ} \mathrm{C}$ for 5 min. DNA was amplified in 35 cycles at $94^{\circ} \mathrm{C}$, Ta $\left({ }^{\circ} \mathrm{C}\right)$, $72^{\circ} \mathrm{C}$ for $45 \mathrm{sec}$ each, followed by a final extension at $72^{\circ} \mathrm{C}$ for $5 \mathrm{~min}$. Ta, annealing temperature $\left({ }^{\circ} \mathrm{C}\right)$.

Table II. Neonatal and maternal characteristics of the IVF, ICSI and spontaneously conceived pregnancies.

\begin{tabular}{|c|c|c|c|c|}
\hline Characteristic & $\begin{array}{l}\text { Spontaneous } \\
\qquad(n=60)\end{array}$ & $\begin{array}{c}\text { IVF } \\
(n=61)\end{array}$ & $\begin{array}{c}\text { ICSI } \\
(n=40)\end{array}$ & $\mathrm{P}$-value \\
\hline Male gender, no. $(\%)$ & $35(58.3)$ & $39(63.9)$ & $19(47.5)$ & NS \\
\hline Twins, no. (\%) & $4(6.7)$ & $26(42.6)$ & $20(50.0)$ & $<0.001$ \\
\hline Gestational age at delivery (week) & $38.8 \pm 1.3$ & $36.3 \pm 2.8^{\mathrm{a}}$ & $37.0 \pm 1.6^{\mathrm{b}}$ & $<0.001$ \\
\hline Preterm delivery $<37$ weeks, no. (\%) & $3(5.0)$ & $24(39.3)$ & $13(32.5)$ & $<0.001$ \\
\hline Birth weight (g) & $3,124 \pm 386$ & $2,554 \pm 631^{\mathrm{a}}$ & $2,785 \pm 451^{b}$ & $<0.001$ \\
\hline Maternal age (year) & $28.2 \pm 4.3$ & $31.7 \pm 4.5^{\mathrm{a}, \mathrm{c}}$ & $28.9 \pm 3.3$ & $<0.001$ \\
\hline
\end{tabular}

IVF, in vitro fertilization; ICSI, intracytoplasmic sperm injection. ${ }^{\mathrm{a} I V F}$ vs. spontaneous, $\mathrm{P}<0.05$; ${ }^{\mathrm{b}} \mathrm{ICSI}$ vs. spontaneous, $\mathrm{P}<0.05$; ${ }^{\mathrm{I}} \mathrm{ICSI}$ vs. IVF, $\mathrm{P}<0.05$.
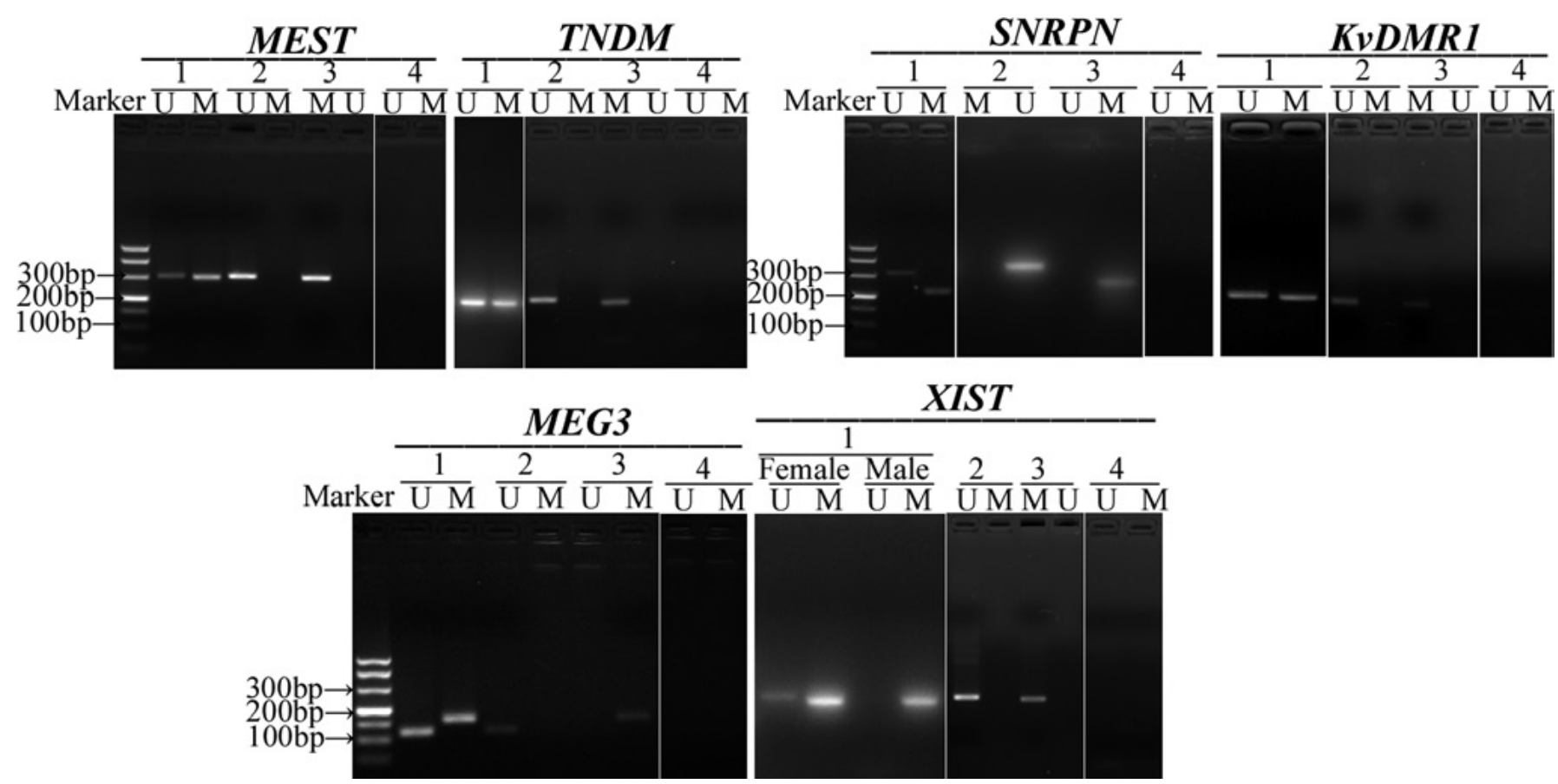

Figure 1. Representative example of methylation-specific PCR (MS-PCR) analysis of six DMRs (MEST, TNDM, SNRPN, KvDMR1, MEG3, XIST) in umbilical cord blood. Lane 1, umbilical cord blood from neonates; lane 2, non-methylated DNA standard was amplified for use as negative control; lane 3, methylated DNA standard was amplified for use as positive control; lane 4, untreated DNA. U, unmethylated-allele-specific products; M, methylated-allele-specific products. 
spontaneous pregnancies showed normal methylation patterns in the six studied DMRs (Fig. 1).

\section{Discussion}

In the present study we found that DNA methylation patterns in children born after ART were identical to the methylation patterns in children conceived spontaneously. Our research indicated a stable methylation status in umbilical cord blood of children regardless of conception mode.

An important concern for infertile couples is to produce a healthy baby; thus, the neonates were objectively and representatively chosen for our study. During gametogenesis and embryogenesis, existing imprints inherited from previous generations are erased, and new imprints are established. Consequently, many studies have focused on the alteration of DNA methylation patterns in gametes and embryos, which are thought to be linked to various steps in ART $(6,28,29)$. However, the number of human oocytes and embryos, to some extent, were too small to be used to investigate the association between offspring after ART and imprinting defects. It is not possible to prove the normal fertilization ability of oocytes when they are used in empirical studies. That is, normal embryos from patients were suitable but not available for ethical reasons. It is plausible to consider that the evaluation of offsprings conceived by ART is the most direct way to evaluate the epigenetic safety of ART compared with the study of human gametes and embryos.

In our study, the clinical data revealed that, compared with the infants conceived spontaneously, infants conceived by ART were more likely to have low birth weight and to be born before term, which is mainly due to the high twinning rate associated with IVF and ICSI (30). Pregnancies by ART have been reported to be associated with an increased risk for adverse perinatal outcomes, including low birth weight, preterm birth and perinatal death. However, a recent study reported that subfertile women conceiving without ART also appeared to be at an increased risk of adverse outcomes, which indicates that these risks should be considered when analyzing the adverse effects of ART (31). In addition, maternal age was higher in the IVF group, which is mainly attributed to unsuccessful attempts to conceive spontaneously. Patients who conceived by ICSI may have begun their treatments earlier due to definite infertility causes and thus had a similar maternal age compared with the patients who conceived spontaneously. The advanced age of infertile couples is also a risk factor for adverse perinatal outcomes (32). Overall, these differences between the groups do not indicate an association between ART and adverse pregnancy outcomes. Moreover, the 3-year clinical follow-up revealed that none of the studied children had clinical symptoms of any imprinting disorders. Hansen et al suggested that most major defects are likely to be detected by 1 year after birth (33). Accordingly, a 3-year follow-up was, to some extent, an adequate time period to provide clinical evidence for evaluating the children studied.

Molecular studies regarding the association between ART and imprinting errors in humans have been primarily carried out using gametes and embryos. Thus, data on offsprings are limited, and the results are inconclusive. Previous studies have revealed that children conceived by ART do not show a higher degree of imprint variability and do not have a higher risk for imprinting disorders $(17,34)$, which were consistent with our results. Rossignol et al suggested that an epigenetic imprinting defect of patients with BWS is not restricted to the $11 \mathrm{p} 15$ region (KCNQ1OT1 and H19), and the involvement of other loci (PEG1/MEST, SNRPN) is also not restricted to patients with BWS (14). Furthermore, several studies have suggested an association between TNDM and disturbance of TNDM DMR (26), human tumors and imprinting defects of MEG3 and XIST $(25,35)$. Taking these data together, our multi-gene study on children born after ART was necessary and reliably assessed the safety of ART.

In the present study, MS-PCR was used to analyze the methylation patterns of imprinted genes, which is a classic strategy for investigating DNA methylation. The accuracy and usefulness of MS-PCR indicates that it can be designed for many DMRs in the genome (36). Our results found normal methylation patterns in six DMRs of the imprinted genes. One related problem of this method includes the mis-amplification of the original untreated DNA with the primers that we used. However, due to the high efficiency of DNA modification with the bisulfite kit, the chance for this error was negligibly small. Indeed, the untreated DNA was not amplified with either primer set in our study (Fig. 1, lane 4). Moreover, Kobayashi et al suggested a difference in the vulnerability of different DMRs to undergo alteration (37). This difference was not detected in our study. However, a slight alteration in the degree of methylation cannot be excluded in our study. Tierling et al observed small changes in methylation of several DMRs in umbilical cord blood, but the authors suggested that the statistical significant differences obtained should be interpreted with caution (17). In contrast, subtle methylation changes cannot be neglected since the accumulation of epigenetic disturbances throughout generations (38), above a certain threshold, may lead to obvious aberrant phenotypes.

A study conducted by Doornbos et al found that an increased incidence of imprinting diseases was associated with increased fertility problems of the parents but not ART (39). Particularly, the intrinsic imprinting defects of spermatozoa appeared to be responsible for the increased incidence of imprinting disorders (37). However, phenotype changes caused by epigenetic disturbances may present at a later stage since the disturbances can be tolerated during development. Meanwhile, an animal study suggested that imprinting errors in fetal germ cells can be transmitted to the next generation (40). Actually, the 32-year period involved in the development of ART is not sufficient to assess the longterm risks linked to epigenetic defects after ART. In additon, the analyzed sample size in our study was far too small to generally exclude rare imprinting disorders. Therefore, a complete safety evaluation may require studies from a twogeneration perspective with a large population.

In conclusion, our results suggest that ART alone is not associated with a significant increase in the methylation variations in imprinted genes in children born after ART. As the impact of epigenetic disturbances at a later stage in the lifespan of humans born as a result of ART is not known, longterm clinical follow-up studies as well as further molecular research of the children born as a result of assisted reproduction are recommended. 


\section{Acknowledgements}

The authors thank all members of the Research Center of Clinical Medicine of Nanfang Hospital for their technical supports and valuable suggestions, and the nurse midwives of Nanfang Hospital for collecting samples for our study. This study was supported by the National Key Basic Research Development Plan of China (973 Program) (2007CB948104).

\section{References}

1. Manipalviratn S, DeCherney A and Segars J: Imprinting disorders and assisted reproductive technology. Fertil Steril 91: 305-315, 2009.

2. Odom LN and Segars J: Imprinting disorders and assisted reproductive technology. Curr Opin Endocrinol Diabetes Obes 17: 517-522, 2010.

3. Jaenisch R and Bird A: Epigenetic regulation of gene expression: how the genome integrates intrinsic and environmental signals. Nat Genet 33 (Suppl): 245-254, 2003.

4. Ferguson-Smith AC and Surani MA: Imprinting and the epigenetic asymmetry between parental genomes. Science 293 1086-1089, 2001.

5. Fauque P, Jouannet P, Lesaffre C, Ripoche MA, Dandolo L, Vaiman D and Jammes H: Assisted Reproductive Technology affects developmental kinetics, H19 Imprinting Control Region methylation and H19 gene expression in individual mouse embryos. BMC Dev Biol 7: 116, 2007.

6. Sato A, Otsu E, Negishi H, Utsunomiya T and Arima T: Aberrant DNA methylation of imprinted loci in superovulated oocytes. Hum Reprod 22: 26-35, 2007.

7. Shi W and Haaf T: Aberrant methylation patterns at the two-cell stage as an indicator of early developmental failure. Mol Reprod Dev 63: 329-334, 2002.

8. Li T, Vu TH, Ulaner GA, et al: IVF results in de novo DNA methylation and histone methylation at an igf2-H19 imprinting epigenetic switch. Mol Hum Reprod 11: 631-640, 2005.

9. Cox GF, Burger J, Lip V, Mau UA, Sperling K, Wu BL and Horsthemke B: Intracytoplasmic sperm injection may increase the risk of imprinting defects. Am J Hum Genet 71: 162-164, 2002.

10. Allen $C$ and Reardon W: Assisted reproduction technology and defects of genomic imprinting. BJOG 112: 1589-1594, 2005.

11. DeBaun MR, Niemitz EL and Feinberg AP: Association of in vitro fertilization with Beckwith-Wiedemann syndrome and epigenetic alterations of LIT1 and H19. Am J Hum Genet 72: 156-160, 2003

12. Maher ER, Brueton LA, Bowdin SC, et al: Beckwith-Wiedemann syndrome and assisted reproduction technology (ART). J Med Genet 40: 62-64, 2003.

13. Halliday J, Oke K, Breheny S, Algar E and Amor DJ: BeckwithWiedemann syndrome and IVF: a case-control study. Am J Hum Genet 75: 526-528, 2004.

14. Rossignol S, Steunou V, Chalas C, et al: The epigenetic imprinting defect of patients with Beckwith-Wiedemann syndrome born after assisted reproductive technology is not restricted to the 11p15 region. J Med Genet 43: 902-907, 2006.

15. Lidegaard O, Pinborg A and Andersen AN: Imprinting diseases and IVF: Danish National IVF cohort study. Hum Reprod 20: 950-954, 2005.

16. Bowdin S, Allen C, Kirby G, et al: A survey of assisted reproductive technology births and imprinting disorders. Hum Reprod 22: 3237-3240, 2007.

17. Tierling S, Souren NY, Gries J, et al: Assisted reproductive technologies do not enhance the variability of DNA methylation imprints in human. J Med Genet 47: 371-376, 2010.

18. Gomes MV, Huber J, Ferriani RA, Amaral NA and Ramos ES: Abnormal methylation at the KvDMR1 imprinting control region in clinically normal children conceived by assisted reproductive technologies. Mol Hum Reprod 15: 471-477, 2009.

19. Kubota T, Das S, Christian SL, Baylin SB, Herman JG and Ledbetter DH: Methylation-specific PCR simplifies imprinting analysis. Nat Genet 16: 16-17, 1997.
20. Zeschnigk M, Lich C, Buiting K, Doerfler W and Horsthemke B: A single-tube PCR test for the diagnosis of Angelman and Prader-Willi syndrome based on allelic methylation differences at the SNRPN locus. Eur J Hum Genet 5: 94-98, 1997.

21. Gomes MV, Gomes CC, Pinto WJ and Ramos ES: Methylation pattern at the KvDMR in a child with Beckwith-Wiedemann syndrome conceived by ICSI. Am J Med Genet A 143: 625-629, 2007.

22. Kosaki K, Kosaki R, Robinson WP, Craigen WJ, Shaffer LG, Sato $\mathrm{S}$ and Matsuo N: Diagnosis of maternal uniparental disomy of chromosome 7 with a methylation specific PCR assay. J Med Genet 37: E19, 2000.

23. White HE, Durston VJ, Harvey JF and Cross NC: Quantitative analysis of SNRPN(correction of SRNPN) gene methylation by pyrosequencing as a diagnostic test for Prader-Willi syndrome and Angelman syndrome. Clin Chem 52: 1005-1013, 2006.

24. Murphy SK, Wylie AA, Coveler KJ, et al: Epigenetic detection of human chromosome 14 uniparental disomy. Hum Mutat 22: 92-97, 2003.

25. Kawakami T, Okamoto K, Ogawa O and Okada Y: XIST unmethylated DNA fragments in male-derived plasma as a tumour marker for testicular cancer. Lancet 363: 40-42, 2004.

26. Mackay DJ, Temple IK, Shield JP and Robinson DO: Bisulphite sequencing of the transient neonatal diabetes mellitus DMR facilitates a novel diagnostic test but reveals no methylation anomalies in patients of unknown aetiology. Hum Genet 116: 255-261, 2005.

27. Al HS, Kupker W, Baschat AA, Sturm R, Bauer O, Diedrich C and Diedrich K: Mini-swim-up: a new technique of sperm preparation for intracytoplasmic sperm injection. J Assist Reprod Genet 12: 428-433, 1995.

28. Borghol N, Lornage J, Blachere T, Sophie GA and Lefevre A: Epigenetic status of the H19 locus in human oocytes following in vitro maturation. Genomics 87: 417-426, 2006.

29. Chen SL, Shi XY, Zheng HY, Wu FR and Luo C: Aberrant DNA methylation of imprinted H19 gene in human preimplantation embryos. Fertil Steril 94: 2356-2358.e1, 2010.

30. Ombelet W, De Sutter P, van der Elst J and Martens G: Multiple gestation and infertility treatment: registration, reflection and reaction-the Belgian project. Hum Reprod Update 11: 3-14, 2005.

31. Jaques AM, Amor DJ, Baker HW, et al: Adverse obstetric and perinatal outcomes in subfertile women conceiving without assisted reproductive technologies. Fertil Steril 94: 2674-2679, 2010.

32. Carolan $\mathrm{M}$ and Frankowska D: Advanced maternal age and adverse perinatal outcome: a review of the evidence. Midwifery 2010 (E-pub ahead of print).

33. Hansen M, Kurinczuk JJ, Bower C and Webb S: The risk of major birth defects after intracytoplasmic sperm injection and in vitro fertilization. N Engl J Med 346: 725-730, 2002.

34. Manning M, Lissens W, Bonduelle M, Camus M, De Rijcke M, Liebaers I and van Steirteghem A: Study of DNA-methylation patterns at chromosome 15q11-q13 in children born after ICSI reveals no imprinting defects. Mol Hum Reprod 6: 1049-1053, 2000.

35. Benetatos L, Dasoula A, Hatzimichael E, Georgiou I, Syrrou M and Bourantas KL: Promoter hypermethylation of the MEG3 (DLK1/MEG3) imprinted gene in multiple myeloma. Clin Lymphoma Myeloma 8: 171-175, 2008.

36. Kubota $\mathrm{T}$, Nonoyama $\mathrm{S}$, Tonoki $\mathrm{H}$, et al: A new assay for the analysis of X-chromosome inactivation based on methylationspecific PCR. Hum Genet 104: 49-55, 1999.

37. Kobayashi H, Hiura H, John RM, et al: DNA methylation errors at imprinted loci after assisted conception originate in the parental sperm. Eur J Hum Genet 17: 1582-1591, 2009.

38. Daxinger L and Whitelaw E: Transgenerational epigenetic inheritance: more questions than answers. Genome Res 20: $1623-1628,2010$.

39. Doornbos ME, Maas SM, McDonnell J, Vermeiden JP and Hennekam RC: Infertility, assisted reproduction technologies and imprinting disturbances: a Dutch study. Hum Reprod 22: 2476-2480, 2007.

40. Anway MD, Cupp AS, Uzumcu M and Skinner MK: Epigenetic transgenerational actions of endocrine disruptors and male fertility. Science 308: 1466-1469, 2005. 